\title{
Zirconium-89-labelled rituximab PET-CT in orbital inflammatory disease
}

\author{
Kamil G. Laban ${ }^{1,2^{*}} \mathbb{D}$, Rachel Kalmann ${ }^{1}$, Roos J. Leguit ${ }^{3}$ and Bart de Keizer ${ }^{4}$
}

\begin{abstract}
Background: Orbital inflammatory diseases are a heterogenic group of conditions that often entail a difficult diagnostic process and many patients are treatment resistant. Inflammatory diseases can be visualized by Zirconium-89-labelled rituximab PET-CT ( ${ }^{89}$ Zr-rituximab PET/CT). In this study, we describe our experience and possible potential of the ${ }^{89} \mathrm{Zr}$-rituximab PET/CT for diagnostic and therapeutic management of refractory orbital inflammation.

Results: Retrospectively, ${ }^{89} \mathrm{Zr}$-rituximab uptake was assessed and related to clinical data. The main outcome measures were the characteristics of the scan and the clinical relation of uptake with the diagnostic process and treatment effectivity. Twelve patients with thyroid eye disease (TED) and suspected idiopathic orbital inflammation (IOI) were scanned. Six patients had a strong ${ }^{89} \mathrm{Zr}$-rituximab uptake and showed a focal distribution within the lesion. Four patients (one TED, three $\mid \mathrm{OI}$ ) responded well to rituximab treatment after a positive scan. ${ }^{89} \mathrm{Zr}$-rituximab PET/CT was essential to the diagnosis of optic nerve meningioma in one patient.

Conclusion: ${ }^{89} \mathrm{Zr}$-rituximab PET/CT has the potential to be a powerful tool for the detection of B cellmediated disease within the orbit and ocular adnexa. This technique can be a valuable addition for diagnosing diseases around the eye and can potentially predict rituximab treatment response in patients with refractory inflammation.
\end{abstract}

Keywords: Idiopathic orbital inflammation, Thyroid eye disease, 89Zr-rituximab PET/CT, Rituximab

\section{Background}

Orbital inflammatory disease comprises of several inflammatory conditions around the eye with different underlying causes [1]. The most common and well-studied cause is thyroid-associated eye disease (TED), while non-thyroid associated orbital inflammation can be a diagnostic challenge and most are considered idiopathic orbital inflammation (IOI) [2, 3]. The most important challenge is the differentiation from malignant entities, especially lymphoid malignancies, because of the grave therapeutic consequences. Currently, the best diagnostic tool available is to perform a biopsy of the orbital process with immunohistochemistry [3]. A biopsy is,

\footnotetext{
* Correspondence: K.G.Laban@umcutrecht.nl

${ }^{1}$ Department of Ophthalmology, University Medical Center Utrecht, Utrecht University, Room E 03.136, P.O. Box 85500, 3508, GA, Utrecht, The Netherlands

${ }^{2}$ Laboratory of Translational Immunology, University Medical Center Utrecht, Utrecht University, Utrecht, The Netherlands

Full list of author information is available at the end of the article
}

unfortunately, not always deemed possible because of deep localization behind the eye and the related risk of complications [4]. From a pathological point of view, biopsies of orbital inflammation often reveal a lymphoplasmacytic infiltrate consisting of (cluster of differentiation (CD) 20+) B cells, CD5+ T cells and polytypical plasma cells. Imaging modalities that can directly detect elements of the pathophysiology of the disease, such as CD20+ B cells, may therefore have potential as an aid in the diagnostic process and management strategies. CD20+ B cell infiltrates have previously been visualized in patients with immune diseases and lymphoma with Zirconium-89-labelled $\left({ }^{89} \mathrm{Zr}\right)$ rituximab positron emission tomography-computed tomography (PET-CT) [5-7]. The use of ${ }^{89} \mathrm{Zr}$-rituximab $\mathrm{PET} / \mathrm{CT}$ in orbital disease has not yet been investigated. Here, we describe our experience and the potential of this technique in aiding in the diagnosis of refractive orbital inflammation. 


\section{Methods}

In this retrospective study, we included 12 patients with an ${ }^{89} \mathrm{Zr}$-rituximab PET/CT in the University Medical Center Utrecht for ophthalmologic pathology. The scans were performed because of suspected orbital inflammatory disease refractory to standard treatment. In five patients, the use of rituximab was considered as alternative treatment and it was given in four patients. At the time of the scan, all patients were rituximab naïve. The standard therapy for IOI and TED consisted of oral prednisone regimen $(60 \mathrm{mg})$ tapered over 3 months or intravenous (IV) methylprednisolone (500-1000 mg/day for 3 days depending on the severity of disease), with the continuation of oral prednisone or IV methylprednisolone regimen in case of insufficient response. Refractory to standard therapy was defined as intolerance, failure to respond to, or inability to taper oral prednisone treatment, the use of multiple IV methylprednisolone regimens or systemic immunosuppressive treatment (adapted from Suhler et al. [8]). IOI was diagnosed by assessing clinical indicators, MRI imaging and an extensive laboratory panel and whenever possible a biopsy [9]. A biopsy for diagnostic confirmation was possible for all IOI except for one IOI located within the orbital apex (case 1). One patient was diagnosed with IgG4-related orbital disease (IgG4+ ROD). The research team investigated clinical data, laboratory workup, and histopathology.

\section{Patients}

From the 12 patients (detailed description in Table 1), 8 were diagnosed with IOI, 2 patients with TED, 1 patient with IgG4+ ROD, and 1 patient ultimately with an optic nerve meningioma. The patients were refractory to standard treatment in the following way: two patients (cases 5 and 6) received oral prednisolone treatment for more than 12 months with inability for tapering. The other 10 patients did not respond to treatment with oral prednisolone and 1 or multiple intravenous (IV) methylprednisolone treatments (range 1-6 IV methylprednisolone regimens). Four patients (cases 2, 3, 11 and 12) were given additional immunosuppressive treatment (either azathioprine $100-150 \mathrm{mg}$, tocilizumab $800 \mathrm{mg}$ or methotrexate $20 \mathrm{mg}$ /week). Both patients with TED did not respond to multiple IV methylprednisolone regimens and retained a clinical activity score (CAS) with a score of four (case 4) and five (case 10). Due to the nature of refractory orbital inflammation, all patients were either under oral prednisolone treatment at the time of the scan or were recently given oral or IV steroids.

\section{${ }^{89}$ Zr-rituximab $\mathrm{PET} / \mathrm{CT}$ procedure}

Seventy-four megabecquerel ${ }^{89}$ Zirconium (with a halflife of $78.4 \mathrm{~h}$ ) was produced and labelled to $10 \mathrm{mg}$ rituximab according to the procedures described previously [10]. No adverse effect occurred on administration of ${ }^{89} \mathrm{Zr}$-rituximab. Three days after intravenous administration, we performed a PET/CT of the head on a TruePoint Biograph mCT40 scanner (Siemens, Erlangen, Germany). We performed a low dose CT scan using Care Dose 4D and Care $\mathrm{kV}$, reference parameters: $40 \mathrm{mAs}, 120 \mathrm{kV}$. Subsequently, the PET was acquired according to the European Association of Nuclear Medicine (EANM) recommendations with a single bed position of $10 \mathrm{~min}$ with the following parameters: PET with time-of-flight and point spread function (TrueX) reconstruction, 4 iterations, 21 subsets, with a filter of

Table 1 Representation of the cases for diagnosis and PET/CT intensity values

\begin{tabular}{|c|c|c|c|c|c|c|c|c|c|c|}
\hline Case & $\begin{array}{l}\text { Final } \\
\text { diagnosis }\end{array}$ & Location & Biopsy & Pain & RAPD & $\begin{array}{l}\text { Proptosis (Hertel } \\
\text { in } \mathrm{mm} \text { ) }\end{array}$ & BCVA & $\begin{array}{l}\text { PET SUVmax } \\
\text { lesion }\end{array}$ & $\begin{array}{l}\text { PET SUVmax bone } \\
\text { marrow }\end{array}$ & $\begin{array}{l}\text { PET SUV max LN } \\
\text { level } 2\end{array}$ \\
\hline 1 & $\mid \mathrm{OI}$ & $\begin{array}{l}\text { Apex with posterior } \\
\text { extension }\end{array}$ & - & Moderate & No & Yes (24-20) & 0.6 & $\begin{array}{l}1.04 \\
\text { (moderate) }\end{array}$ & 2.07 & 11.36 \\
\hline 2 & $|O|$ & Myositis & + & Severe & No & $Y_{e s}^{a}$ & 1.0 & 0.68 (low) & 2.01 & 7.97 \\
\hline 3 & $|O|$ & Lacrimal gland & + & Moderate & No & No & 1.2 & 3.88 (high) & 5.32 & 11.32 \\
\hline 4 & TED & Pan-myositis & - & None & No & Yes (23-23) & 1.0 & 0.33 (low) & 2.91 & 5.45 \\
\hline 5 & $\operatorname{lgG} 4+$ & Myositis & + & Mild & No & Yes (16-24) & 1.2 & $\begin{array}{l}1.58 \\
\text { (moderate) }\end{array}$ & 4.43 & 8.90 \\
\hline 6 & $|O|$ & Diffuse mass & + & Moderate & No & $Y_{e s}^{a}$ & 0.9 & 3.11 (high) & 3.73 & 15.87 \\
\hline 7 & $|O|$ & Diffuse mass & + & Moderate & No & $Y_{e s}^{a}$ & 1.0 & 2.12 (high) & 3.27 & 14.45 \\
\hline 8 & Meningioma & Apex & - & None & Yes & No & 0.6 & 0.79 (low) & 2.49 & 5.81 \\
\hline 9 & $|O|$ & Diffuse mass & + & Severe & Yes & No & 0.5 & 3.82 (high) & 3.64 & 12.12 \\
\hline 10 & TED & Pan-myositis & - & Mild & No & Yes (29-29) & 0.7 & 3.47 (high) & 4.07 & 12.10 \\
\hline 11 & $|O|$ & Myositis & + & Severe & No & No & 0.9 & 4.24 (high) & 3.52 & 13.71 \\
\hline 12 & $|O|$ & Myositis & + & Severe & No & No & 1.0 & 0.68 (low) & 4.83 & 19.15 \\
\hline
\end{tabular}

$I O I=$ idiopathic orbital inflammation; $T E D=$ thyroid eye disease; $R A P D=$ relative afferent pupillary defect; $B C V A=$ best-corrected visual acuity

${ }^{a}$ Clinical and radiological proptosis, not quantified with Hertel 
$7.5 \mathrm{~mm}$ full width at half maximum [11]. We used tonsillar, submandibular, submental, pre- and post-auricular, and occipital lymph nodes as a positive control of CD20+ (B cell) targeting. For standardized uptake value (SUV) measurements, we used the lean body mass-corrected formula. We regarded a quantification of the PET-positivity (maximal SUV) above 2.0 to be a strong positivity, consistent with the PET-positivity of lymph nodes and bone marrow in the head and neck area, and a maximum SUV between 1.0 and 2.0 as moderate uptake.

\section{Results}

\section{PET-CT analysis}

In six patients, we found a high ${ }^{89} \mathrm{Zr}$-rituximab PET uptake (standardized uptake value $(\mathrm{SUV})>2.0$ ) within the orbital masses (Table 1, example in Fig. 1a). One patient with a strong PET uptake had an active TED with CAS 5. In two patients, a moderate ${ }^{89} \mathrm{Zr}$-rituximab uptake was seen, of which one patient had an IOI in the orbital apex and one patient was diagnosed with IgG4+ ROD (Fig. 1b). In four patients, there was no pathological ${ }^{89} \mathrm{Zr}$-rituximab uptake. We diagnosed patients with a negative scan as myositis (two patients), active TED with CAS 4, and meningioma in the orbital apex (case 8) which became most likely after a positive Gallium-68-labelled DOTA-TATE PET/CT (Fig. 2b), as meningiomas usually have a high somatostatin receptor expression [12]. In this last patient, a concurrent sinusitis of the right maxillary sinus coincidentally showed a strong
${ }^{89} \mathrm{Zr}$-rituximab uptake (Fig. 2c). Interestingly, IOI located in the lacrimal gland or diffuse within the orbit had strong uptake of ${ }^{89} \mathrm{Zr}$-rituximab, whilst myositis and masses of the apex had moderate to no uptake. Strong ${ }^{89} \mathrm{Zr}$-rituximab uptake was inhomogeneous with a focal intense uptake within the lesion.

\section{Histopathology}

Eight patients had an open incisional biopsy through a lid crease or swinging-eyelid surgical approach, dependent on the localization within the orbit [3]. For all but one (case 5), the histological images were not suggestive for IgG4-related disease. Histopathological examination in seven biopsies showed signs of a chronic inflammation with a lymphoplasmacytic infiltrate, characterized by both $\mathrm{CD} 20+\mathrm{B}$ cells and $\mathrm{CD} 3+\mathrm{T}$ cells CD138+ plasma cells had a polytypic lambda and kappa distribution and either a predominant IgG or IgA differentiation for orbital and lacrimal gland tissue, respectively. In one biopsy, we only found fatty tissue without inflammation due to sampling error and a deep orbital localization.

\section{Rituximab treatment}

Four patients were treated with rituximab $(2 \times 1000 \mathrm{mg}$ IV, MabThera, Roche) after a positive ${ }^{89} \mathrm{Zr}$-rituximab PET/CT. All treated patients showed improvement of complaints within the first weeks after treatment. This effect was temporary in 3 out of 4 cases as some of the complaints returned to lesser extent. In one case, all

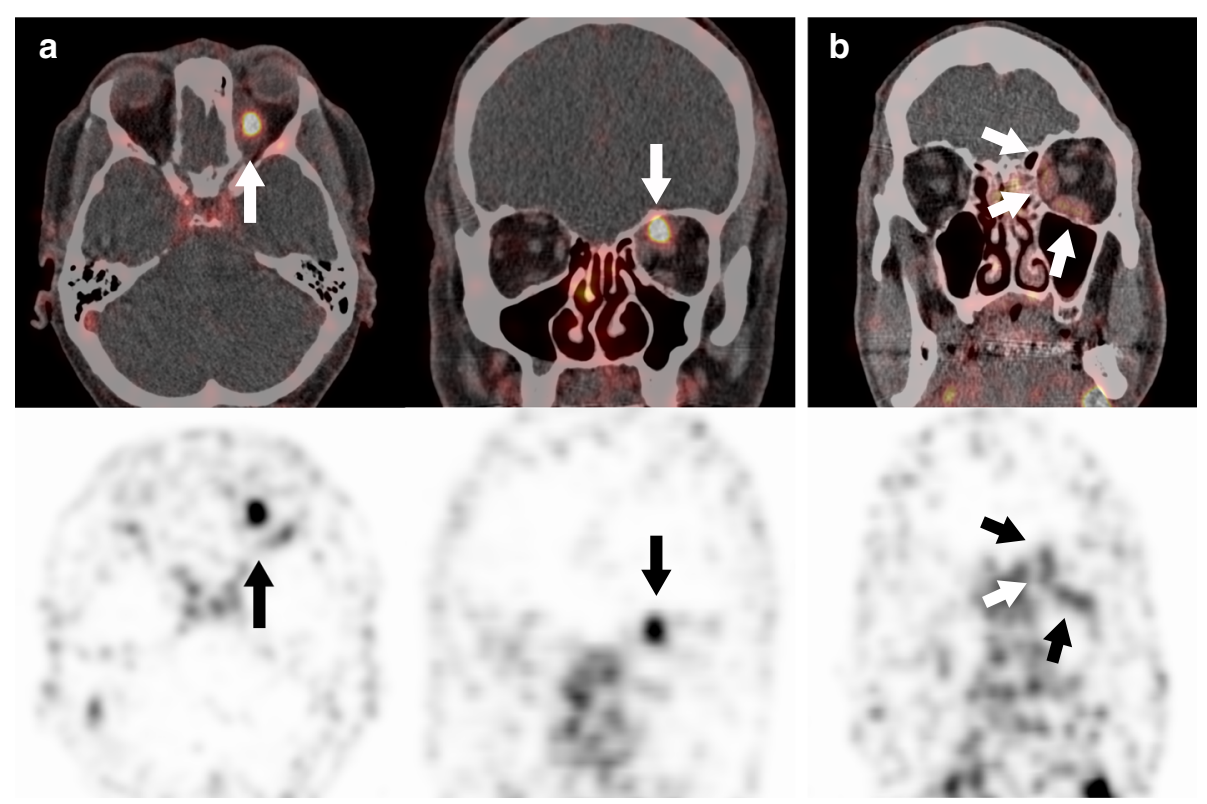

Fig. 1 Examples of 89Zr-rituximab PET/CT uptake. a Strong uptake in fusion image and PET-only image for the axial and coronal planes (arrows point at the lesion, SUVmax> 2.0; case 6). b Moderate uptake in fusion image and PET-only image (arrows point at affected muscles, SUVmax 1.02.0, case 5) 

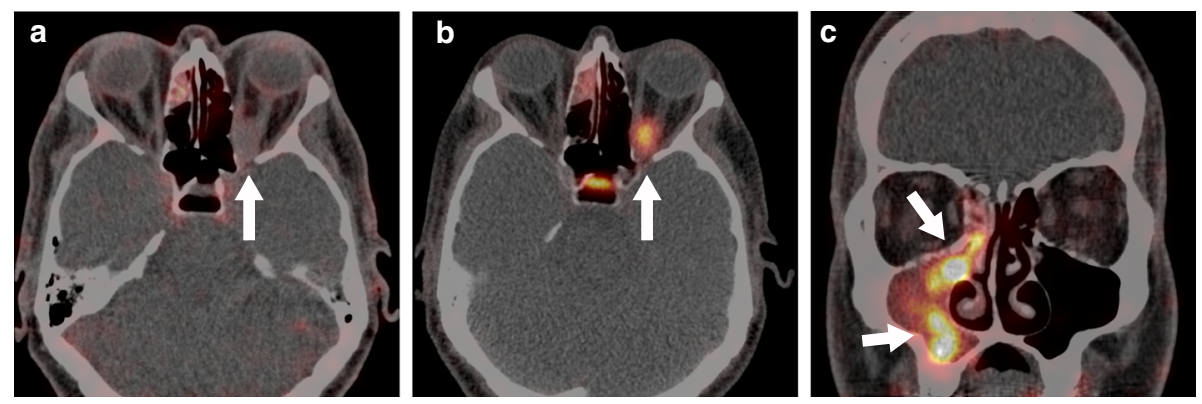

Fig. 2 Patient with a meningioma in the left orbital apex and sinusitis of the right maxillary sinus (case 8). a Negative ${ }^{89} \mathrm{Zr}$-rituximab PET/CT (white arrow, SUVmax <1.0). b Positive 68Ga DOTA-TATE PET/CT, white arrow. $\mathbf{c}^{89}$ Zr-rituximab uptake in a co-existing sinusitis at the contralateral side, white arrows

complaints were initially gone (vision $>20 / 20$, no pain and no diplopia), but some complaints returned 6 months later (case 7). A second rituximab treatment was given after 6 months and the patient responded well with remission of complaints. Three other cases (case 9-11) had a significant reduction of complaints (improvement of visual acuity to $20 / 20$, mild or no pain and improvement of eye motility). Of these, one received a second rituximab dose 4 months later (case 10), one had a relapse after 2 months and received additional treatment of oral prednisone (15 mg) and azathioprine (150 $\mathrm{mg}$ ) (case 11), and one is currently monitored over 6 months whilst still improving (case 9). Two patients had an MRI scan after treatment with rituximab and both showed major radiological improvement with almost complete regression of the mass (case 7 and 9 Fig. 3a-d).

\section{Other treatments}

Two patients with a positive scan did not receive rituximab treatment. One patient improved after treatment with oral prednisone and receiving infliximab for coexisting Crohn's disease (case 3) and the other patient improved on IV steroids (case 6). The latter patient was previously treated with oral prednisone for more than 12 months and had a biopsy after ${ }^{89} \mathrm{Zr}$-rituximab PET/ CT was performed. With a confirmed diagnosis of IOI, this patient was therefore first treated with IV steroids. Patients with a negative scan were not treated with rituximab and had stable disease, refractory to treatment. The patient with IgG4+ ROD (case 5) had a good response after additional IV steroids were given.

\section{Discussion}

We describe our experience of ${ }^{89} \mathrm{Zr}$-rituximab PET/CT in 12 patients suspected of refractory orbital inflammation within the University Medical Center Utrecht. We have found a strong ${ }^{89} \mathrm{Zr}$-rituximab uptake in orbital inflammatory diseases of the lacrimal gland and as a mass or diffuse within the orbit. Idiopathic myositis and involvement of the orbital apex showed ${ }^{89} \mathrm{Zr}$-rituximab uptake to a lesser extent. A focal density was found in masses with a strong uptake. All four patients treated with rituximab after a positive ${ }^{89} \mathrm{Zr}$-rituximab PET/CT had a good response during one or multiple treatments.

Five studies previously investigated the use of ${ }^{89} \mathrm{Zr}$-rituximab PET/CT in humans with different disease entities. Two studies investigated B cell lymphoma in a total of 11 patients [7, 13]. Lymphoma masses showed ${ }^{89} \mathrm{Zr}$-rituximab PET uptake that was greater in the tumour mass without a preload of unlabelled rituximab [7]. The tumour uptake of labelled rituximab correlated with the in-tissue CD20 expression [13]. One study investigated the predictive value of ${ }^{89} \mathrm{Zr}$-rituximab PET/ $\mathrm{CT}$ for the effectiveness of rituximab treatment in rheumatoid arthritis patients [5]. A case report described the use of ${ }^{89} \mathrm{Zr}$-rituximab PET/CT in the diagnostic process of neurolymphomatosis in the sciatic nerve [6]. Finally, one study investigated three patients with multiple sclerosis, reporting no penetration of ${ }^{89} \mathrm{Zr}$-rituximab in the brain [14].

Besides Zirconium-89, intact CD20 labelling with rituximab has been performed with Iodine-124 [15] for patients with rheumatoid arthritis and Technetium-99m [16] in several inflammatory conditions, showing feasibility for CD20 imaging. However, Zirconium-89 remains the most suitable for internalizing intact monoclonal antibodies [17]. ${ }^{89} \mathrm{Zr}$-rituximab has a relatively long half-life and high effective dose of approximately $0.5 \mathrm{mSv} / \mathrm{MBq}$ [18]. The radiation dose should therefore be considered and balanced to the clinical benefits.

Surgical (open) biopsies are recommended for the diagnosis of orbital masses [3]. Unfortunately, biopsies deep in the orbit can be difficult, not-representative and potentially lead to severe complications to the optic nerve and extra-ocular muscles [4, 19]. The ${ }^{89} \mathrm{Zr}$-rituximab PET/CT can be of aid in distinguishing inflammatory and lymphoproliferative disorders from other orbital diseases, as we demonstrate in case 8 (Fig. 2). This technique can, therefore, in combination with 

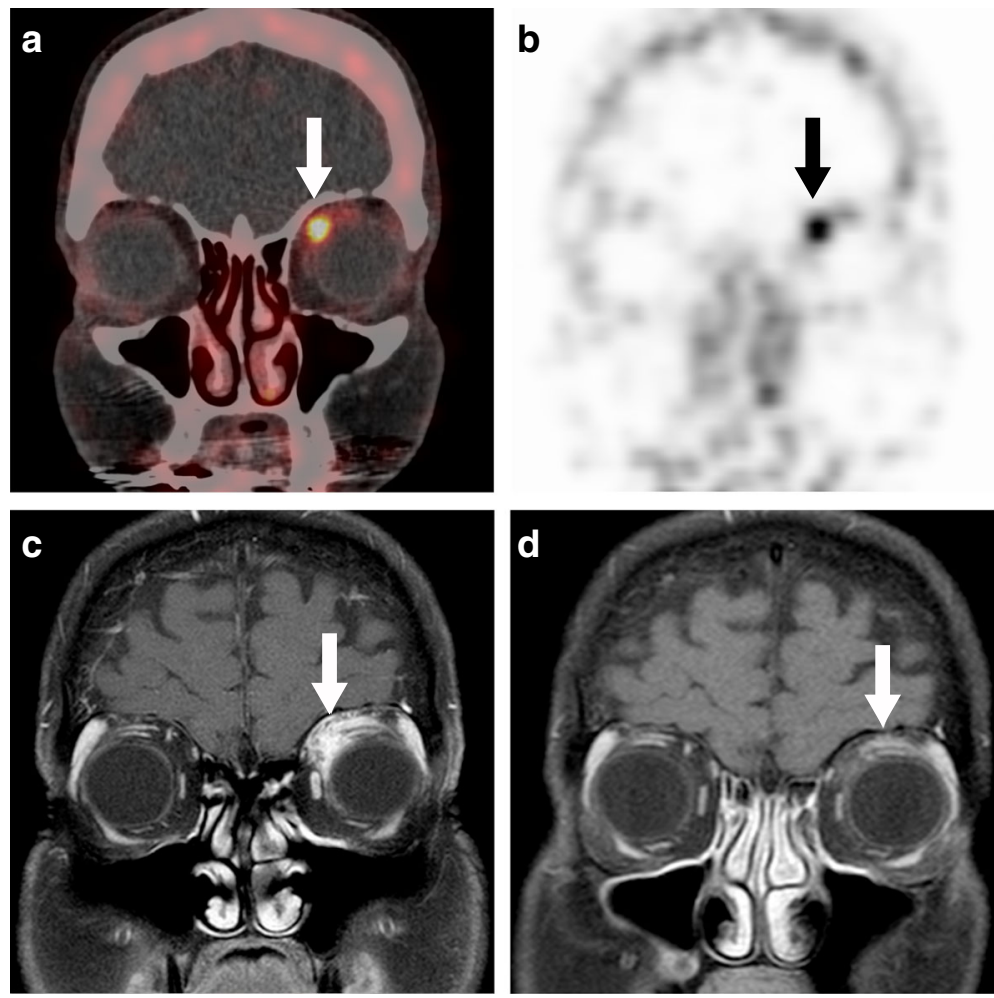

Fig. 3 Rituximab treatment response (case 7). a ${ }^{89}$ Zr-rituximab PET/CT fusion image with focal uptake in the lesion, white arrow points at the lesion. b PET only image, black arrow points at the lesion. Initial (c) and post-treatment MRI at 3 months after treatment (d), illustrating treatment response

clinical and laboratory findings [9] and MRI imaging [20], have additional value for a comprehensive diagnosis in difficult cases.

Our study shows that stronger focal ${ }^{89} \mathrm{Zr}$-rituximab intensity can occur within the orbital mass (Fig. 3). It was previously suggested that a higher tumour $\mathrm{CD} 20+$ expression correlated with a higher $\mathrm{PET} / \mathrm{CT}$ intensity in lymphoma patients [13]. In our opinion, representable biopsies should yield the densest area of inflammatory cells within the tumour to provide the most information and exclude lymphoma. The yield of the inflammatory area within the biopsies was dependent on the morphology of the inflammation within the normal tissue as well as the depth of the mass within the orbit, reflecting the difficulty of an orbital biopsy. Because we show focal uptake in this study, we believe that the ${ }^{89} \mathrm{Zr}$-rituximab PET/CT could be used as pre-biopsy orientation for targeting higher intensity areas during incisional biopsies for difficult cases.

The role of rituximab as a treatment in refractive orbital inflammatory disease is currently under investigation. Previous case reports and a phase I/II trial have indicated a strong potential for the effectiveness of rituximab treatment in refractory orbital inflammation $[8,21]$. However, not all patients benefit from this treatment, and the non-responders have an unnecessary exposure to potentially severe adverse effects. For IOI, the presence of a mixed $\mathrm{B}$ cell and $\mathrm{T}$ cell profile in the histopathological analysis of the masses reflects the involvement of both cells in the pathogenesis of the disease [22]. Although theoretically logical, there is no evidence of better rituximab effectivity in IOI patients with a more profound B cell involvement. In TED, varying results have been published of the effectiveness of rituximab treatment [23-27]. Most early reports and a randomized controlled trial [24] describe clinical improvement with treatment in almost all patient, while another randomized controlled trial did not show an overall improvement compared to placebo. The search for factors that can predict the effectiveness of rituximab in TED and other orbital inflammatory diseases continues [28].

The use of ${ }^{89} \mathrm{Zr}$-rituximab PET/CT to predict rituximab treatment response has been demonstrated in a small cohort of rheumatoid arthritis patients by quantification of the uptake [5]. Although limited by the number of patients, we can now extrapolate this theory to orbital inflammatory diseases as we see a good response of rituximab in high-uptake patients in the current 
study. We would encourage future research investigating the predictive potential of rituximab therapy in inflammatory diseases, including TED, to use ${ }^{89} \mathrm{Zr}$-rituximab $\mathrm{PET} / \mathrm{CT}$ as an objective and measurable tool.

Several limitations of this study exist, inherent to the retrospective nature. We were not able to include patients diagnosed with a biopsy-proven orbital lymphoma. We would expect a high uptake in lymphoma patients $[7,10]$ and a comparison with inflammatory orbital conditions is warranted for the potential for differentiation. Also, not all patients were treated with rituximab, including the patients with a negative scan. We could therefore not compare patients with a positive and negative scan for treatment effectivity.

\section{Conclusion}

This study describes our institutional experience with the ${ }^{89} \mathrm{Zr}$-rituximab PET/CT in orbital inflammatory diseases. This technique has the potential to be a powerful tool for the detection of $\mathrm{B}$ cell-mediated disease within the orbit and ocular adnexa. By visualizing the CD20+ B cells, it can be a valuable addition to the diagnostic armamentarium for orbital inflammatory disease. Higher focal intensities within an orbital mass were found that can potentially pinpoint the location for more representative surgical biopsies. Patients with a strong ${ }^{89} \mathrm{Zr}$-rituximab PET/CT uptake responded well to rituximab treatment. We encourage further research of this technique in the diagnostic process and to predict rituximab treatment response for orbital inflammatory diseases.

\section{Abbreviations \\ ${ }^{89}$ Zr-rituximab PET/CT: Zirconium-89-labelled rituximab positron emission tomography/computed tomography; CAS: Clinical activity score; CD: Cluster of differentiation; IgG4 + ROD: IgG4+-related orbital disease; IOI: Idiopathic orbital inflammation; IV: Intravenous; TED: Thyroid-associated eye disease}

\section{Acknowledgements}

Not applicable

\section{Authors' contributions}

$K G L, R K$ and BdK devised the study and handled the clinical and radiological data. RJL handled the pathological data. KGL, RK, RJL and BdK interpreted the data and constructed the manuscript. All authors read and approved the manuscript in final form.

\section{Funding}

No funding was received for this study.

\section{Availability of data and materials}

The datasets generated during and/or analysed during the current study are available from the corresponding author on reasonable request.

\section{Ethics approval and consent to participate}

We performed this study in compliance with the 1964 Helsinki Declaration and its later amendments and federal laws in the Netherlands and the European Union. Following review of the study design and protocol, the local Medical Research Ethical Committee (MREC)(Medisch Ethische Toetsingscommissie Utrecht) confirmed that the Medical Research Involving Human Subjects Act (WMO) does not apply to this study and that therefore an official approval of this study by the local MREC is not required under the
WMO (protocol number: 18-120). All patients signed written informed consent for participation in this study.

\section{Consent for publication}

All patients signed written informed consent for the use of their data in this article.

\section{Competing interests}

The authors declare that they have no competing interests.

\section{Author details}

${ }^{1}$ Department of Ophthalmology, University Medical Center Utrecht, Utrecht University, Room E 03.136, P.O. Box 85500, 3508, GA, Utrecht, The Netherlands. ${ }^{2}$ Laboratory of Translational Immunology, University Medical Center Utrecht, Utrecht University, Utrecht, The Netherlands. ${ }^{3}$ Department of Pathology, University Medical Center Utrecht, Utrecht University, Utrecht, The Netherlands. ${ }^{4}$ Department of Radiology and Nuclear Medicine, University Medical Center Utrecht, Utrecht University, Utrecht, The Netherlands.

Received: 13 May 2019 Accepted: 12 July 2019

Published online: 30 July 2019

\section{References}

1. Shields JA, Shields CL, Scartozzi R. Survey of 1264 patients with orbital tumors and simulating lesions: the 2002 Montgomery lecture, part 1. Ophthalmology. 2004;111:997-1008.

2. Gordon LK. Orbital inflammatory disease: a diagnostic and therapeutic challenge. Eye. 2006;20:1196-206.

3. Mombaerts I, Rose GE, Garrity JA. Orbital inflammation: biopsy first. Surv Ophthalmol. 2016:61:664-9.

4. Dagi Glass LR, Freitag SK. Orbital inflammation: corticosteroids first. Surv Ophthalmol. 2016;61:670-3.

5. Bruijnen S, Tsang-A-Sjoe M, Raterman H, Ramwadhdoebe T, Vugts D, van Dongen $\mathrm{G}$, et al. B-cell imaging with zirconium-89 labelled rituximab PET-CT at baseline is associated with therapeutic response 24 weeks after initiation of rituximab treatment in rheumatoid arthritis patients. Arthritis Res Ther. 2016;18:266.

6. de Jong A, Mous R, van Dongen GAMS, Hoekstra OS, Nievelstein RAJ, de Keizer B. (89) Zr-rituximab PET/CT to detect neurolymphomatosis. Am J Hematol. 2016;91:649-50.

7. Muylle K, Flamen P, Vugts DJ, Guiot T, Ghanem G, Meuleman N, et al. Tumour targeting and radiation dose of radioimmunotherapy with $90 Y$-rituximab in CD20+ B-cell lymphoma as predicted by 89Zr-rituximab immuno-PET: impact of preloading with unlabelled rituximab. Eur J Nucl Med Mol Imaging. 2015;42: 1304-14.

8. Suhler EB, Lim LL, Beardsley RM, Giles TR, Pasadhika S, Lee ST, et al. Rituximab therapy for refractory orbital inflammation: results of a phase $1 / 2$, dose-ranging, randomized clinical trial. JAMA Ophthalmol. 2014;132:572-8.

9. Mombaerts I, Bilyk JR, Rose GE, McNab AA, Fay A, Dolman PJ, et al. Consensus on diagnostic criteria of idiopathic orbital inflammation using a modified Delphi approach. JAMA Ophthalmol. 2017;135:769-76.

10. Verel I, Visser GWM, Boellaard R, Stigter-van Walsum M, Snow GB, van Dongen GAMS. 89Zr immuno-PET: comprehensive procedures for the production of 89Zr-labeled monoclonal antibodies. J Nucl Med. 2003:44: 1271-81.

11. Boellaard R, Delgado-Bolton R, Oyen WJG, Giammarile F, Tatsch K, Eschner W, et al. FDG PET/CT: EANM procedure guidelines for tumour imaging: version 2.0. Eur J Nucl Med Mol Imaging. 2015:42:328-54.

12. Klingenstein A, Haug AR, Miller C, Hintschich C. Ga-68-DOTA-TATE PET/CT for discrimination of tumors of the optic pathway. Orbit. 2015;34:16-22.

13. Jauw YWS, Zijlstra JM, de Jong D, Vugts DJ, Zweegman S, Hoekstra OS, et al. Performance of 89Zr-labeled-rituximab-PET as an imaging biomarker to assess CD20 targeting: a pilot study in patients with relapsed/refractory diffuse large B cell lymphoma. PLoS One. 2017;12:e0169828 Glod JW, editor.

14. Hagens MH, Killestein J, Yaqub MM, van Dongen GA, Lammertsma AA, Barkhof F, et al. Cerebral rituximab uptake in multiple sclerosis: $A^{89}$ Zr-immunoPET pilot study. Mult Scler J. 2018;24:543-5.

15. Tran L, Huitema ADR, van Rijswijk MH, Dinant HJ, Baars JW, Beijnen JH, et al. CD20 antigen imaging with 124l-rituximab PET/CT in patients with rheumatoid arthritis. Hum Antibodies. 2011;20:29-35. 
16. Malviya G, Anzola KL, Podestà E, Laganà B, Del Mastro C, Dierckx RA, et al. (99m)Tc-labeled rituximab for imaging B lymphocyte infiltration in inflammatory autoimmune disease patients. Mol Imaging Biol. 2012;14: 637-46.

17. van Dongen GAMS, Poot AJ, Vugts DJ. PET imaging with radiolabeled antibodies and tyrosine kinase inhibitors: immuno-PET and TKI-PET. Tumour Biol. 2012;33:607-15 Springer.

18. Jauw YWS, Menke-van der Houven van Oordt CW, Hoekstra OS, Hendrikse NH, Vugts DJ, Zijlstra JM, et al. Immuno-positron emission tomography with Zirconium-89-labeled monoclonal antibodies in oncology: what can we learn from initial clinical trials? Front Pharmacol. 2016;7:131 Frontiers Media SA.

19. Chastain JB, Sindwani R. Anatomy of the orbit, lacrimal apparatus, and lateral nasal wall. Otolaryngol Clin N Am. 2006;39:855-64.

20. Hiwatashi A, Togao O, Yamashita K, Kikuchi K, Kamei R, Yoshikawa H, et al. Diffusivity of intraorbital lymphoma vs. inflammation: comparison of single shot turbo spin echo and multishot echo planar imaging techniques. Eur Radiol. 2018;28:325-30

21. Abell RG, Patrick A, Rooney KG, McKelvie PA, McNab AA. Complete resolution of idiopathic sclerosing orbital inflammation after treatment with rituximab. Ocul Immunol Inflamm. 2015;23:176-9.

22. Mombaerts I, Schlingemann RO, Goldschmeding R, Koornneef L. Idiopathic granulomatous orbital inflammation. Ophthalmology. 1996;103:2135-41.

23. Stan MN, Garrity JA, Carranza Leon BG, Prabin T, Bradley EA, Bahn RS. Randomized controlled trial of rituximab in patients with Graves' orbitopathy. J Clin Endocrinol Metab. 2015:100:432-41.

24. Salvi M, Vannucchi G, Currò N, Campi I, Covelli D, Dazzi D, et al. Efficacy of B-cell targeted therapy with rituximab in patients with active moderate to severe Graves' orbitopathy: a randomized controlled study. J Clin Endocrinol Metab. 2015;100:422-31.

25. Khanna D, Chong KKL, Afifiyan NF, Hwang CJ, Lee DK, Garneau HC, et al. Rituximab treatment of patients with severe, corticosteroid-resistant thyroidassociated ophthalmopathy. Ophthalmology. 2010;117:133-139.e2.

26. Silkiss RZ, Reier A, Coleman M, Lauer SA. Rituximab for thyroid eye disease Ophthalmic Plast Reconstr Surg. 2010;26:310-4.

27. El Fassi D, Nielsen CH, Hasselbalch HC, Hegedüs L. Treatment-resistant severe, active graves' ophthalmopathy successfully treated with B lymphocyte depletion. Thyroid. 2006;16:709-10.

28. Stan MN, Salvi M. MANAGEMENT OF ENDOCRINE DISEASE: rituximab therapy for Graves' orbitopathy-lessons from randomized control trials. Eur J Endocrinol. 2017;176:R101-9.

\section{Publisher's Note}

Springer Nature remains neutral with regard to jurisdictional claims in published maps and institutional affiliations.

\section{Submit your manuscript to a SpringerOpen ${ }^{\odot}$ journal and benefit from:}

- Convenient online submission

- Rigorous peer review

- Open access: articles freely available online

- High visibility within the field

- Retaining the copyright to your article

Submit your next manuscript at $\boldsymbol{\nabla}$ springeropen.com 